Supplementary Information:

\title{
The Molecular Mechanism of Iron(III) Oxide
}

\section{Nucleation}

Johanna Scheck ${ }^{l}$, Baohu $W^{l, 2}$, Markus Drechsler ${ }^{3}$, Rose Rosenberg ${ }^{l}$, Alexander E. S. Van

Driessche $^{4}$,Tomasz M. Stawski ${ }^{5,6}$ and Denis Gebauer ${ }^{1 *}$

1. Department of Chemistry, Physical Chemistry, University of Konstanz, Universitätsstr. 10, D78457 Konstanz, Germany

2. Jülich Centre for Neutron Science, Outstation at the MLZ, Forschungszentrum Jülich, Lichtenbergstr. 1, D-85748 Garching, Germany

3. Laboratory for Soft Matter Electron Microscopy, BIMF; University of Bayreuth, Universitätsstr. 30, D-95440 Bayreuth, Germany

4. ISTerre, CNRS, F-38041 Grenoble, France

5. Cohen Geochemistry, School of Earth and Environment, University of Leeds, LS2 9JT, Leeds, UK

6. German Research Centre for Geosciences, GFZ, 14473, Potsdam, Germany 


\section{Experimental}

All chemicals were used as received: iron(III)choride hexahydrate (Sigma Aldrich, puriss. p.a., ACS reagent, 98.0 - 102\%), hydrochloric acid (Merck, $0.1 \mathrm{M}$ ), sodium hydroxide (Merck, $0.1 \mathrm{M}$ ), sodium chloride (VWR, 99.9\%), nitric acid (Sigma Aldrich, puriss. p.a., reag. ISO, reag. Ph. Eur., $\geq 65 \%$ ). All solutions and dilutions were prepared with water of Milli-Q quality if not stated otherwise. All samples were prepared freshly and analyzed immediately after drawing them from the titration. The hydrolysis was performed with an automated commercially available titration setup provided by Metrohm (Filderstadt, Germany). The titration device (836 Titrando), which operates two dosing units (800 Dosino) is operated with custom-made software (Tiamo 2.3) that controls the dosing of the solutions. The dosing units allow dosing in small steps of down to $0.2 \mu \mathrm{L}$ through burette tips, which are equipped with special valves preventing the diffusion of solution into the titration reservoir. $\mathrm{pH}$ values were measured with a double-junction $\mathrm{pH}$ electrode (Metrohm, 6.0269.100). The electrode was calibrated regularly using three $\mathrm{pH}$ buffers (Mettler-Toledo). Turbidity measurements were carried out using an optrode sensor (6.1115.000) with a $610 \mathrm{~nm}$ laser. The titrations were carried out at ambient temperature in $100 \mathrm{~mL}$ beakers. After every titration the glassware and the electrodes were cleaned with water of Milli-Q quality. In the titration experiments a solution of $0.1 \mathrm{M} \mathrm{FeCl}_{3} \times 6$ $\mathrm{H}_{2} \mathrm{O}$ in $0.1 \mathrm{M} \mathrm{HCl}$ was added at a rate of $0.01 \mathrm{~mL} / \mathrm{min}$ into $25 \mathrm{~mL}$ of $0.01 \mathrm{M}$ hydrochloric acid that was adjusted to the desired $\mathrm{pH}$ value by addition of the required amount of $0.05 \mathrm{M} \mathrm{NaOH}$ prior to the titration. This $\mathrm{pH}$ value was kept constant throughout the titration by counter titration of $0.05 \mathrm{M}$ sodium hydroxide solution. AUC was conducted on an Optima XL-I (Beckman-Coulter, Palo Alto, CA). The samples were centrifuged at $60,000 \mathrm{rpm}$ at $25^{\circ} \mathrm{C}$ and the Rayleigh interference optics were used for analysis. The experiments were performed in $12 \mathrm{~mm}$ titanium double sector cells (Nanolytics, Potsdam, Germany). $10 \mathrm{mM}$ hydrochloric acid was used as reference solution. Evaluation of the data was carried out using the software SEDFIT by Schuck ${ }^{1}$ applying Lamm equation modelling for 1-4 non-interacting species to determine sedimentation and diffusion coefficients as well as concentration of up to 4 species in a solution. Sedimentation coefficient distributions were obtained by using SEDFIT and the density calculated with sedimentation coefficients from AUC experiments and sizes from SAXS. For cryo-TEM, samples taken at different times from the titration were spread on lacey carbon filmed copper grids by blotting with a filter paper. The resulting thin film was vitrified by quickly plunging the grids into liquid ethane at its freezing point. Specimens were examined at temperatures around $90 \mathrm{~K}$ with a Zeiss/LEO EM922 Omega TEM. Collected images were processed with a background-subtraction routine and, where appropriate, a smoothing filter (Butterworth Filter) was applied to reduce noise. The size distribution was obtained using ImageJ, whereby the lower limit for the particle analysis was set to a particle area of $0.25 \mathrm{~nm}^{2}$. With this limit, 1540 species were included in the determination of the size distribution. UV-Vis spectra were obtained in situ utilizing a PEEK flow cell coupled to a spectrometer (Ocean Optics, Dunedin, USA). Conductivity measurements were performed using a conductivity cell (Metrohm, 6.0910.120) connected to a conductivity module 856 (Metrohm, 2.856.0010). The cell constant was determined prior to the measurements using a $100 \mu \mathrm{S} / \mathrm{cm}$ conductivity standard solution 
(Metrohm, 6.2324.110). Samples for SAXS analysis were measured in a sampledetector distance of $2.33 \mathrm{~m}$ and with a x-ray wavelength of $0.1 \mathrm{~nm}$ to cover the q-range ( $q$ is the scattering vector, $q=(4 \pi / \lambda) \sin (\theta / 2), \theta$ is the scattering angle) from 0.07 to $4 \mathrm{~nm}^{-}$

${ }^{1}$. The used detector was a Quantum 210R CCD detector from ADSC. A peristaltic pump (Gilson, Middleton, WI, MiniPuls 3 ) was used to continuously transport small amounts of the reaction solution at a flow rate of $\sim 10 \mathrm{~mL} / \mathrm{s}$ from the titration system into a custombuilt PEEK flow-through cell with a Kapton capillary and back into the system. The capillary exhibits a wall thickness of $\sim 10 \mu \mathrm{m}$ and an internal diameter of $1.5 \mathrm{~mm}$. The scattering patterns were obtained with $30 \mathrm{~s}$ exposure time for all time stages. This approach provides time-resolved in situ analyses of the hydrolysis reaction. The SAXS patterns were normalized to an absolute scale and azimuthally averaged to obtain the intensity profiles, and the solvent background was subtracted. The angular scale was calibrated using the scattering peaks of silver behenate. For references, the scattering patterns of the empty capillary and the capillary filled with water were recorded. Analyses of the SAXS data are described in more detail in the Supplementary Information, section 4. Zeta potential measurements were conducted with a Zetasizer Nano ZS (Malvern Instruments, Worcestershire, UK) at $25^{\circ} \mathrm{C}$ with clear disposable zeta cells.

\section{Titrations at varying iron concentrations}

The titration experiments provide homogeneous reaction conditions due to slow mixing and low addition rates of the reaction partners. Addition rates as well as the concentrations of the added solutions were chosen to ensure homogeneous mixing and accessibility of the distinct stages. However, it is also important to minimize dilution effects and realize experiments on a time scale of a few hours. To investigate the influence of mixing and confirm the applicability of the chosen settings, titrations were carried out with different iron addition rates. As the utilized addition rate of $10 \mu \mathrm{L} / \mathrm{min}$ is the slowest one that is technically possible, the concentration of the iron salt solution was lowered and thus the net addition rate of the amount of iron ions. Figure S1 shows the titration data obtained for different addition rates and different concentrations of the iron solution at $\mathrm{pH} 2.85$. The titration curves display generally the same shape and a similar slope in the linear regime at higher iron(III) concentrations. For very low concentrations of the added iron(III) solution the transition in the titration curve to the linear regime seems to appear earlier than for higher concentrations. However, under these experimental conditions, significant dilution effects arise from the fact that high volumes of the iron(III) solution have to be added to increase the concentration of iron(III) ions in the titration solution. A titration carried out at high addition rates shows a trend in the opposite direction, suggesting that the addition rate has a slight influence on the concentration range of iron(III) ions, at which the transition in the curves occurs, but does not influence the overall shape or the final slope of the titration curve. The settings that were chosen in this work (red graph) lie in the same range of those carried out at significantly lower concentrations. There are only minor differences in the titration curves induced by a change in the addition rates of the amount of iron(III) ions, which are 
mostly due to dilution effects; it can be concluded that the experimental settings used in this work are representative for the investigated system.

\section{Determination of equilibrium constants}

The titration data for the early stage of the titration provides information on the equilibrium underlying the hydrolysis reaction. The initial progression can be represented by a linear asymptote, which was added to the data by eye (see Figure S2). Undoubtedly, this line corresponds to the equilibrium yielding $\mathrm{Fe}(\mathrm{OH})^{2+}$ that can be characterized by equilibrium constant $\mathrm{K}_{1}$ according to ${ }^{2}$ :

$$
K_{1}=\frac{\left[\mathrm{Fe}(\mathrm{OH})^{2+}\right]\left[\mathrm{H}^{+}\right]}{\left[\mathrm{Fe}^{3+}\right]}
$$

The $\mathrm{H}^{+}$concentration is given by the $\mathrm{pH}$ value of the solution, while $\left[\mathrm{Fe}(\mathrm{OH})^{2+}\right.$ ] can be obtained by calculating the amount of consumed hydroxide ions represented by single points on the added asymptotes. Accordingly, the concentration of free iron(III) ions can be calculated by subtracting the amount of reacted (asymptotical) hydroxides from the added iron(III) ion concentration. The values obtained for $\mathrm{K}_{1}$ at the investigated $\mathrm{pH}$ values are compiled in Table S1. The value of $\log \mathrm{K}_{1}$ of $-3.09 \pm 0.16$ (main value \pm standard deviation, $\mathrm{N}=7$, ionic strength of about $0.02 \mathrm{M}$ ) is larger than the value for $\log \mathrm{K}_{1}$ of -2.2 reported in the literature at similar ionic strengths, and is actually closer to the values between -2.96 and -3.05 that were obtained for an ionic strength of $3 \mathrm{M}^{2-4}$

The law of mass action for the determination of $\mathrm{K}_{1}$ (equation 1) is based on the equilibrium $\left(\mathrm{FeH}_{2} \mathrm{O}\right)^{3+} \rightleftharpoons(\mathrm{FeOH})^{2+}+\mathrm{H}^{+}$. Here, the equilibrium $\mathrm{H}^{+}$concentration is considered to result from iron(III) hydrolysis. However, a low $\mathrm{pH}$ level is in fact realized by the addition of $\mathrm{HCl}$. From the point of view of the principle of LeChatelier, this assures that the equilibrium is shifted towards the Fe(III) aquo complex, i.e. mitigating hydrolysis. Since $\mathrm{Fe}(\mathrm{III})$ is highly acidic, hydrolysis takes place at very low $\mathrm{pH}$ values and low iron concentrations. This, in turn, categorically implies that the iron(III) hydroxo complex is highly stable, however, $\mathrm{K}_{1} \ll 1$ (Table S1), and consequently $\Delta \mathrm{G}_{1}=-\mathrm{RT} \ln \mathrm{K}_{1}>0$. This contradiction is due to the flawed mass balance of equation 1 , when considered individually, i.e. that much more protons are actually present than generated based on the corresponding equilibrium. This can be resolved when accounting for the (very low) hydroxide concentration providing a realistic mass balance, i.e. $\mathrm{Fe}^{3+}+\mathrm{OH}^{-} \rightleftharpoons(\mathrm{FeOH})^{2+}$. This reaction yields:

$$
K_{2}=\frac{\left[\mathrm{Fe}(\mathrm{OH})^{2+}\right]}{\left[\mathrm{Fe}^{3+}\right]\left[\mathrm{OH}^{-}\right]}
$$

The equilibrium concentrations of the different species can be calculated as described above, while $\left[\mathrm{OH}^{-}\right]$is calculated from the measured $\mathrm{pH}$ value based upon the selfdissociation of water. Consistently, $\mathrm{K}_{2}>>1$ and $\Delta \mathrm{G}_{1}<0$ (Table S1), in accord with the expected, distinct thermodynamic stability of the formed iron(III) hydroxo complex. 
Note that the titration-based data give analytical concentrations that do not account for activity. Nevertheless, the values for $\mathrm{K}_{1}$ and $\mathrm{K}_{2}$ do not show a distinct trend with $\mathrm{pH}$.

\section{Determination of the transition region at all investigated $\mathrm{pH}$ levels}

From the experiments, in which the iron(III) addition was stopped, a transition region for the titration at $\mathrm{pH} 2.4$ can be determined as outlined in the main text. It is found that the transition occurs in the region, where the titration profiles (for continuous iron(III) addition) start to significantly bend upwards. To locate this transition region and enable a correspondent identification of this region for the other $\mathrm{pH}$ values, without the need to carry out tedious stop experiments for all of them, the titration data was fitted with a polynomial function to avoid potential artifacts arising from the noise of the data. The first derivative of this fitted function shows a distinct behavior that can be recognized for all investigated $\mathrm{pH}$ values (Figure S3). This enables the localization of the region, i.e. the range of iron concentrations at which the transition occurs for all investigated $\mathrm{pH}$ values (Supplementary Table S2). For the titration at $\mathrm{pH} 2.05$, no transition occurs within experimental duration.

\section{SAXS experiments}

\section{Titration for SAXS experiments}

Figure S4 shows the titration data of the experiment that was performed for the SAXS experiment. The shape of this curve can be compared to the titrations described for the other experiments (Figure 1). The onset of aggregation that was observed in the scattering patterns is located just prior to the region that was identified as the transition region, in which the reaction mechanism changes.

\section{General remarks}

Small-angle X-ray scattering (SAXS) is a very useful technique to study the structure (shape, size) of scattering entities and their interaction in solution. The scattering intensity, I(q), for a monodisperse system at a scattering angle of $\theta$ with scattering vector $q=(4 \pi / \lambda) \sin (\theta / 2)$, can be expressed as

$$
I(q)=\frac{N}{V}(\Delta \rho)^{2} V_{p}^{2} P(q) S(q)
$$

where N/V is the number of particles per unit volume in the solution, $V_{p}$ is the volume of a single particle, and $(\Delta \rho)^{2}$ is the scattering contrast (for X-rays; the square of the difference in electron density between the scattering species and the solvent). $P(q)$ is the form factor of the scattering objects that relates to their shape and size. Usually, in dilute, non-interacting systems, the scattering curves show a form factor behavior. $S(q)$ is the structure factor, which contains information about the interactions between the 
scattering objects. The structure factor $S(q)$ can be extracted by dividing the total scattering intensity I(q) by the form factor of the sample (Figure $3 b$ ).

The repulsive structure factor, which quantifies the primary species interactions/correlations, is included in our fitting process using the "Hayter-MSAStructure" model $\left.\right|^{5-6}$. The model is based on screened electrostatic interactions between charged particles in the presence of counter-ions and the amount of salt added to the solution. This accounts for a repulsive screened Coulomb interaction potential, which has only been used for the samples in the stages earlier than $3300 \mathrm{~s}$. The model is defined by the volume fraction of the hard sphere, the particle charge, the diameter of particles and the ionic strength, temperature and dielectric constant of solvent. The data was fitted using an Igor Pro based small-angle scattering analysis software developed by the National Institutes of Standard and Technology (NIST, USA). ${ }^{7}$

The repulsive structure factor evolution, fitted by employing the "Hayter-MSA-Structure" model on the early stages of the reaction is shown in Figure S7. Results show that after $1800 \mathrm{~s}$ the structure factor can be well fitted by the model. The interaction strength becomes lower with increasing ionic strength (Figure S7). The surface charge of the primary species is very high (above 60 elementary charges) especially for the early times (Table S3). These unrealistically high charges show that a model of electrostatic stabilization for explaining repulsive interactions between the scattering species is implausible. This is confirmed by the fact that zeta potential measurements performed on the reaction solution in the pre-nucleation stage $(\mathrm{pH} \mathrm{2.5,550} \mathrm{s)} \mathrm{yielded} \mathrm{a} \mathrm{zeta}$ potential of $21.5 \pm 1.5 \mathrm{mV}$ (main value \pm standard deviation, $\mathrm{N}=6$ ). Generally, zeta potentials above $30 \mathrm{mV}$ can be assigned to electrostatic stabilized nanoparticle suspensions. ${ }^{8}$ Note that the sizes of the spheres obtained from the fit are inherent to the model assumption, i.e. to explain the measured structure factors by charged spheres. In the light of their unrealistic high charge, also the obtained sizes between 0.5 and $1.2 \mathrm{~nm}$ do not necessarily reflect realistic proportions.

\section{Determination of particle size distributions from SAXS curves}

SAXS scattering curves were analyzed by using a Monte Carlo method in order to get information on the size distribution of the particles. Monte-Carlo data fitting was performed using the McSAS software as described by Bressler ${ }^{9}$. In this method, the SAXS pattern is first simulated and compared to the experimental curve. The advantage of this method is that one does not need to assume a particular shape for the size distribution of the particles. The only assumptions needed are the shape of the particles and the absence of inter-particle interactions that would otherwise determine the appearance of a structure factor peak in the SAXS curves. In the time regime later than $5400 \mathrm{~s}$, the power law exponents change from 1 to 2, indicating Gaussian-chain like structure formation. Thus a Gaussian-chain model was used to describe the scattering curves. The form factor of a single chain corresponds to a Gaussian chain in solution whose scattering function can be described by the Debye function ${ }^{10}$ : 


$$
P(q)=2 /\left(q^{2} R_{g}^{2}\right)^{2}\left[e^{-q^{2} R_{g}^{2}}-1+q^{2} R_{g}^{2}\right]
$$

where $R_{g}$ is the radius of gyration of the chain.

Figure S6 shows the fit results and the corresponding radius of gyration distributions weighted by the volume at different times after $5400 \mathrm{~s}$. At $5400 \mathrm{~s}$, most of the scattering arises from aggregates of primary species, whose radius of gyration is around $3 \mathrm{~nm}$. The size distribution then becomes broader, and mean sizes larger than $8 \mathrm{~nm}$ start to appear at $10800 \mathrm{~s}$. As the formation of the large aggregates proceeds, the maximum of the size distribution does not shift toward larger sizes and the volume fraction becomes large.

\section{SAXS data reduction and analysis}

SAXS experiments were performed at the non-crystalline diffraction beamline BL11NCD at the ALBA in Barcelona, Spain. Samples were measured in a sample-detector distance of $2.33 \mathrm{~m}$ and $X$-ray wavelength of $0.1 \mathrm{~nm}$ to cover the $\mathrm{q}$ range from 0.07 to 4 $\mathrm{nm}^{-1}$ ( $\mathrm{q}$ is the scattering vector, $\mathrm{q}=(4 \pi / \lambda) \sin (\theta / 2), \theta$ is the scattering angle).

A peristaltic pump (Gilson MiniPuls 3 ) was used to continuously transport small amounts of the reaction solution at a flow rate of $\sim 10 \mathrm{~mL} / \mathrm{s}$ from the titration system into a custom-built PEEK flow-through cell with a Kapton capillary and back into the system. The capillary exhibits a wall thickness of $\sim 10 \mu \mathrm{m}$, and an internal diameter of $1.5 \mathrm{~mm}$. The scattering patterns were obtained with $30 \mathrm{~s}$ exposure time for all time stages. This approach provides time-resolved in situ analyses of the hydrolysis reaction. The SAXS patterns were normalized to an absolute scale and azimuthally averaged to obtain the intensity profiles, and the solvent background was subtracted. The angular scale was calibrated using the scattering peaks of silver behenate. For reference, the scattering patterns of the empty capillary and the capillary filled with a mixture of $\mathrm{HCl}$ and $\mathrm{NaOH}$ at the same ratio as at the end of the titration experiment were recorded.

For SAXS experiments on solution samples measured in a capillary with a very thin wall, water and solvent are also measured in the same capillary with the same instrument configuration. The final scattering intensity of the sample is:

$$
I(q)=\frac{\frac{I(q)_{s}}{t_{s} T_{s}}-\frac{I(q)_{b}}{t_{b} T_{b}}}{\left.\left(\frac{I_{w}}{t_{w} T_{w}}-\frac{I_{E C}}{t_{E C} T_{E C}}\right)\right|_{\operatorname{lnm}^{-1} \leqslant \mathrm{Q} \leqslant 4 \mathrm{~nm}^{-1}}} \cdot 0.0164
$$

Where $I(q)_{s}, I(q)_{b}, I(q)_{w}$, and $I(q)_{E C}$ are the measured intensities of sample, solution background, water and empty capillary, respectively. The bottom term in the high $\mathrm{q}$ range is a flat scattering ( $q$ independent), whereas $T$ is the transmission and $t$ the irradiation time. 


\section{Calculation of density from gyration radius}

To obtain the density from SAXS and AUC data, the gyration radius $R_{G}$ from the scattering experiments has to be converted to the hydrodynamic diameter $\mathrm{d}_{\mathrm{H}}$. The shape of the objects can be assumed to be a hard sphere. In the case of hard spheres, the hydrodynamic diameter can be calculated using the following equation ${ }^{11}$ :

$$
d_{H}=2 \sqrt{\frac{5}{3}} \cdot R_{G}
$$

For polymeric species the relationship is as follows ${ }^{11}$ :

$$
d_{H}=2 \cdot \frac{R_{G}}{1.3}
$$

From the SAXS experiments, a gyration radius of $3 \mathrm{~nm}$ and therefore a hydrodynamic diameter of $7.7 \mathrm{~nm}$ for hard spheres and $4.6 \mathrm{~nm}$ for polymeric species was found. The relationship between the hydrodynamic diameter obtained by SAXS and the sedimentation coefficient $s$ from AUC experiments for calculating the density of the species is:

$$
d_{h}=\sqrt{\frac{18 \cdot \eta_{0} \cdot s}{\rho_{i}-\rho_{0}}}
$$

where $\eta_{0}$ is the viscosity and $\rho_{0}$ the density of the solvent, and $\rho_{\mathrm{i}}$ the density of the sedimenting species. ${ }^{12}$

\section{Influence of ionic strength on the hydrolysis}

To investigate whether the non-aggregative behavior of the species observed in the equilibrium stages of the reaction is due to electrostatic stabilization, a series of titration experiments with varying ionic strength was carried out. To increase the ionic strength, different amounts of $\mathrm{NaCl}$ were added to the starting solution. Figure $\mathrm{S} 9$ compares titration data of the original experiment with hydrolysis experiments at higher ionic strengths. For an initial ionic strength of $98 \mathrm{mM}$, compared to $8 \mathrm{mM}$ in the original experiment, the curve shows nearly the same behavior. Further increase of the $\mathrm{NaCl}$ content up to an ionic strength of $253 \mathrm{mM}$ leads to a decrease in base consumption in the early stages while for the more advanced hydrolysis reaction it exhibits a steeper slope than in the original experiment. However, the change in reaction mechanism characterized by the upwards-bent, which clearly coincides with the onset of aggregation as determined by SAXS, is -if anything - delayed when the ionic strength is increased. Thus, the data show clearly that the species that arise in the beginning of the reaction are not destabilized by an increase in ionic strength, as it would be expected for electrostatic stabilization of nanoparticles. This is also confirmed by the findings from the 
repulsive structure factor fitting that is described in chapter 4 of the Supplementary Information. The decrease in base consumption indicates that less hydroxo ligands are bound with increasing ionic strength, which is due to a decreased activity. Then, after phase separation, the reaction is accelerated in comparison, which may then indeed be assigned to screening of the electrostatic stabilization of the particles of the newly formed phase.

\section{Evaluation of free iron concentration based on conductivity measurements}

The conductivity measurements carried out during the titrations provide the overall conductivity of the solution with all contributing species such as ions and charged species. The theoretical composition of the reaction mixture is accessible throughout the entire experiment. Assuming the formation of iron(oxy)hydroxides, it is thus possible to calculate the conductivity $\mathrm{K}_{\mathrm{x}}$ arising from the dissolved ions (all other than iron ions) using their concentration $c_{x}$ and specific conductivity $\lambda_{x}$ (taken from ${ }^{13}$ ).

$$
K_{x}=c_{x} * \lambda_{x}
$$

The remaining deviation from the measured value can be assigned to free iron ions.

$$
K_{\text {meas }}-\left(K_{\mathrm{H}^{+}}+K_{C l^{-}}+K_{\mathrm{Na}^{+}}\right)=K_{\mathrm{Fe}^{3+}}
$$

The cell constant of the conductivity cell was determined prior to the experiments and used by the program for data correction. This constant is highly temperature dependent and can change with time as passivation or deformations have a high impact on its value. To ensure that for each experiment the right cell constant was used, a correction was carried out. This was accomplished by comparing the calculated with the measured values for the conductivity at the beginning of the titration prior to the addition of any solutions. At this time point these values ought to be equal as all concentrations are known and no chemical reaction has occurred. The quotient of calculated and measured value provides the factor by which the whole data set has to be corrected in order to avoid a systematic error. These corrected values were used to determine the conductivity of the iron ions and therewith the concentration of free iron ions in the reaction solution was obtained using equation (7) (Figure S9).

For a $\mathrm{pH}$ value of 2.01 for which no hydrolysis was observed, the calculated iron concentration exceeds the theoretical value. This can be explained by the loss of electrolyte from the $\mathrm{pH}$ electrode. During the measurement $3 \mathrm{M} \mathrm{KCl}$ diffuses through the porous membrane of the electrode, increasing the conductivity of the electrode. This is an inevitable issue. However, additional ions in the solution increase the conductivity and it can be therefore concluded that the observed effect of iron ions being bound actually exists and the effect on the conductivity is diminished by the leakage of the $\mathrm{pH}$ electrode. Another uncertainty is the molar conductivity of iron ions taken from literature. ${ }^{13-14}$ This value describes the molar conductivity obtained from iron ions in aqueous solutions at $25^{\circ} \mathrm{C}^{14}$. At the native $\mathrm{pH}$ value of water, hydrolysis of the iron(III) solution occurs. Thus, the species for which conductivities were determined have to be 
rather conceived as partly hydrolysed complexes than as iron ions, even for low concentrations.

Nevertheless, even though the analytical iron concentration remains inaccessible due to the leakage of electrolyte and inaccessibility of the molar conductivity of the iron ions, the difference between the added and the measured iron ion concentration is bound to be credited to a reduction in the conductivity caused by the formation of species contributing less or not at all to the overall conductivity. At earlier times and for very low $\mathrm{pH}$ values, however, the measured conductivity is in line with the value expected for the free ions in solution. Hence, these measurements corroborate the formation of a separated phase containing iron ions in accordance to the experiments described above.

\section{References}

(1) Schuck, P., Size-Distribution Analysis of Macromolecules by Sedimentation Velocity Ultracentrifugation and Lamm Equation Modeling. Biophys. J. 2000, 78, 1606-1619.

(2) Hedström, B. O. A., Studies on the Hydrolysis of Metal Ions VII. The Hydrolysis of the Iron(III) Ion, $\mathrm{Fe}^{3+}$. Arkiv För Kemi 1952, 6, 1-16.

(3) Flynn, C. M., Hydrolysis of Inorganic Iron(III) Salts. Chem. Rev. 1984, 84, 31-41.

(4) Baes, C. F.; Mesmer, R. S., The Hydrolysis of Cations. Wiley-VCH: Weinheim, 1977; Vol. 81.

(5) Hansen, J.-P.; Hayter, J. B., A Rescaled MSA Structure Factor for Dilute Charged Colloidal Dispersions. Mol. Phys. 1982, 46, 651-656.

(6) Hayter, J. B.; Penfold, J., An Analytic Structure Factor for Macroion Solutions. Mol. Phys. 1981, 42, 109-118.

(7) Kline, S. R., Reduction and Analysis of SANS and USANS Data Using IGOR Pro. J. Appl. Crystallogr. 2006, 39, 895-900.

(8) Goldstein, N.; Greenlee, L. F., Influence of Synthesis Parameters on Iron Nanoparticle Size and Zeta Potential. J. Nanopart. Res. 2012, 14, 1-15.

(9) Bressler, I.; Pauw, B. R.; Thunemann, A. F., McSAS: Software for the Retrieval of Model Parameter Distributions from Scattering Patterns. J. Appl. Crystallogr. 2015, 48, 962-969.

(10) Glatter, O. K., O., Small Angle X-ray Scattering. Academic Press: London, 1982.

(11) Harding, S. E., On the Hydrodynamic Analysis of Macromolecular Conformation. Biophys. Chem. $1995,55,69-93$.

(12) Mächtle, W.; Börger, L., Analytical Ultracentrifugation of Polymers and Nanoparticles. Springer Berlin Heidelberg: Berlin, Heidelberg, 2006.

(13) Lide, D. R.; Haynes, W. M., CRC Handbook of Chemistry and Physics: A Ready-Reference Book of Chemical and Physical Data. CRC: Boca Raton, Fla, 2009.

(14) Milazzo, G., Electrochemistry: Theoretical Principles and Practical Applications. Elsevier Pub. Co.: 1963. 


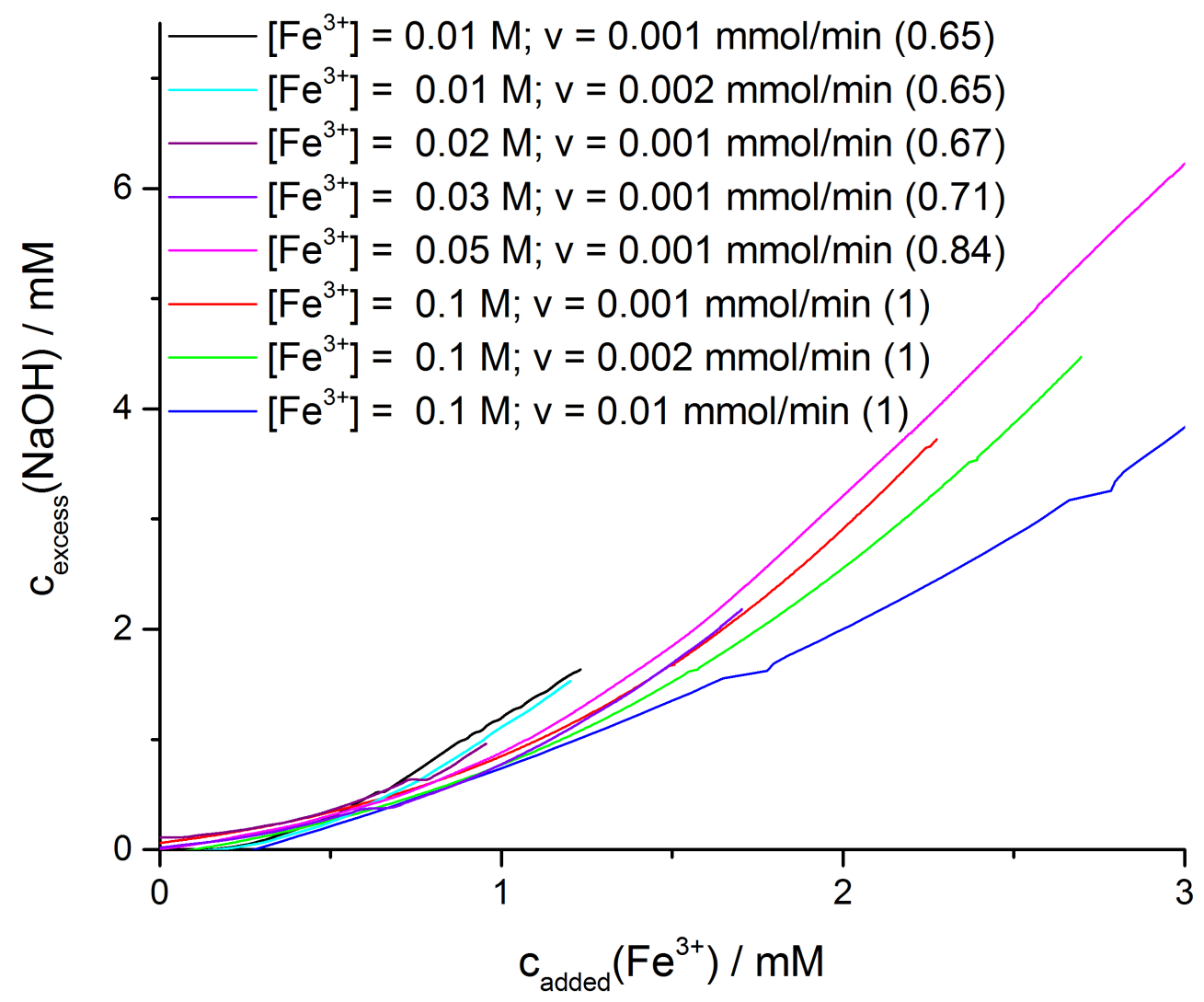

Figure S1 | Titration curves obtained at different addition rates for the amount of iron(III). The experimental conditions used in this work are represented by the red graph. The values in parentheses in the legend represent the dilution factor relative to this rate. For explanation see chapter 1 in the SI. The data shown in this figure was smoothed. 


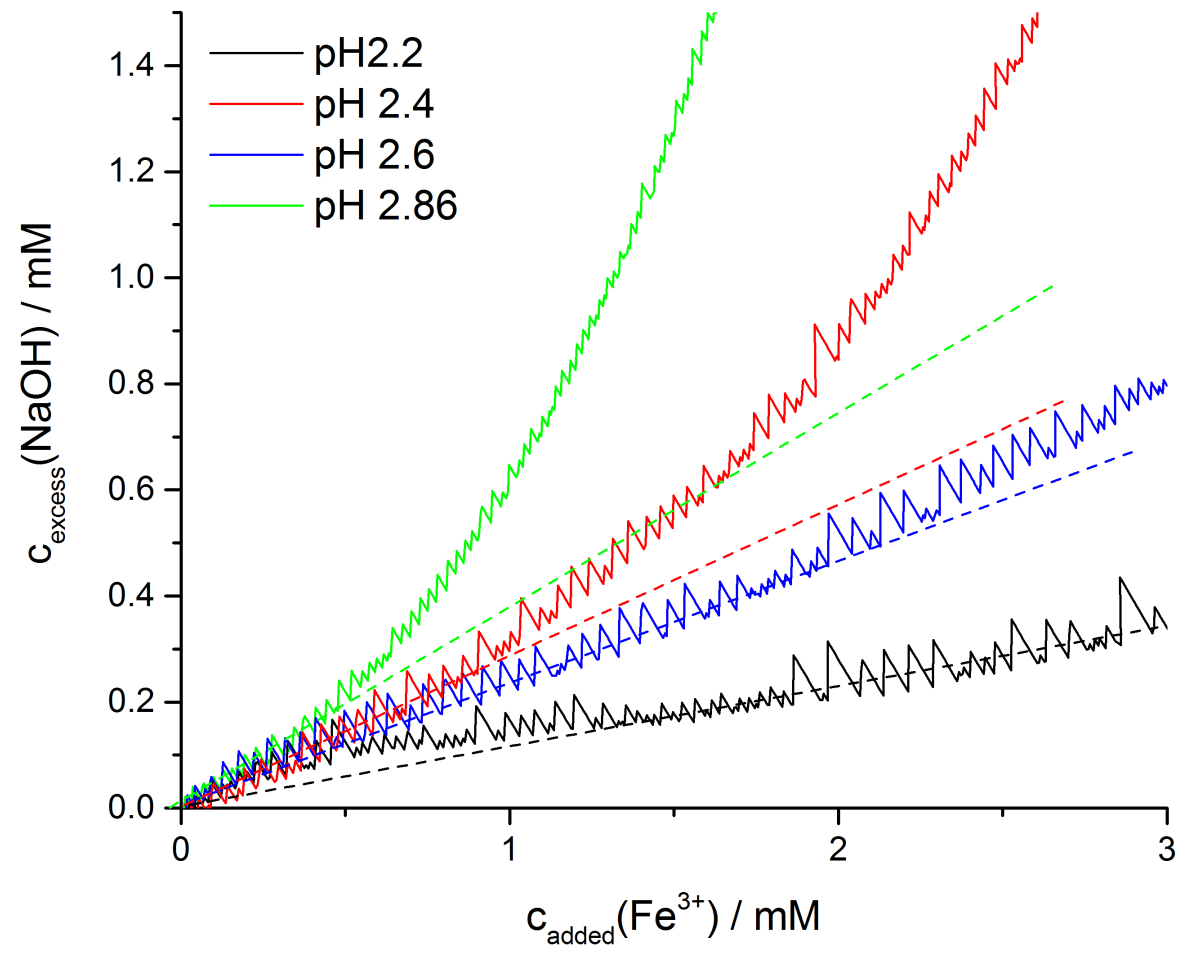

Figure S2 | Calculation of - $\log \mathrm{K}$ in the initial stage of the reaction. Enlarged view of selected titration curves (raw data) from Figure 1. The dashed lines are asymptotes corresponding to the equilibrium concentration of $\mathrm{Fe}(\mathrm{OH}) 2+$ giving the equilibrium constants compiled in Table S1. 


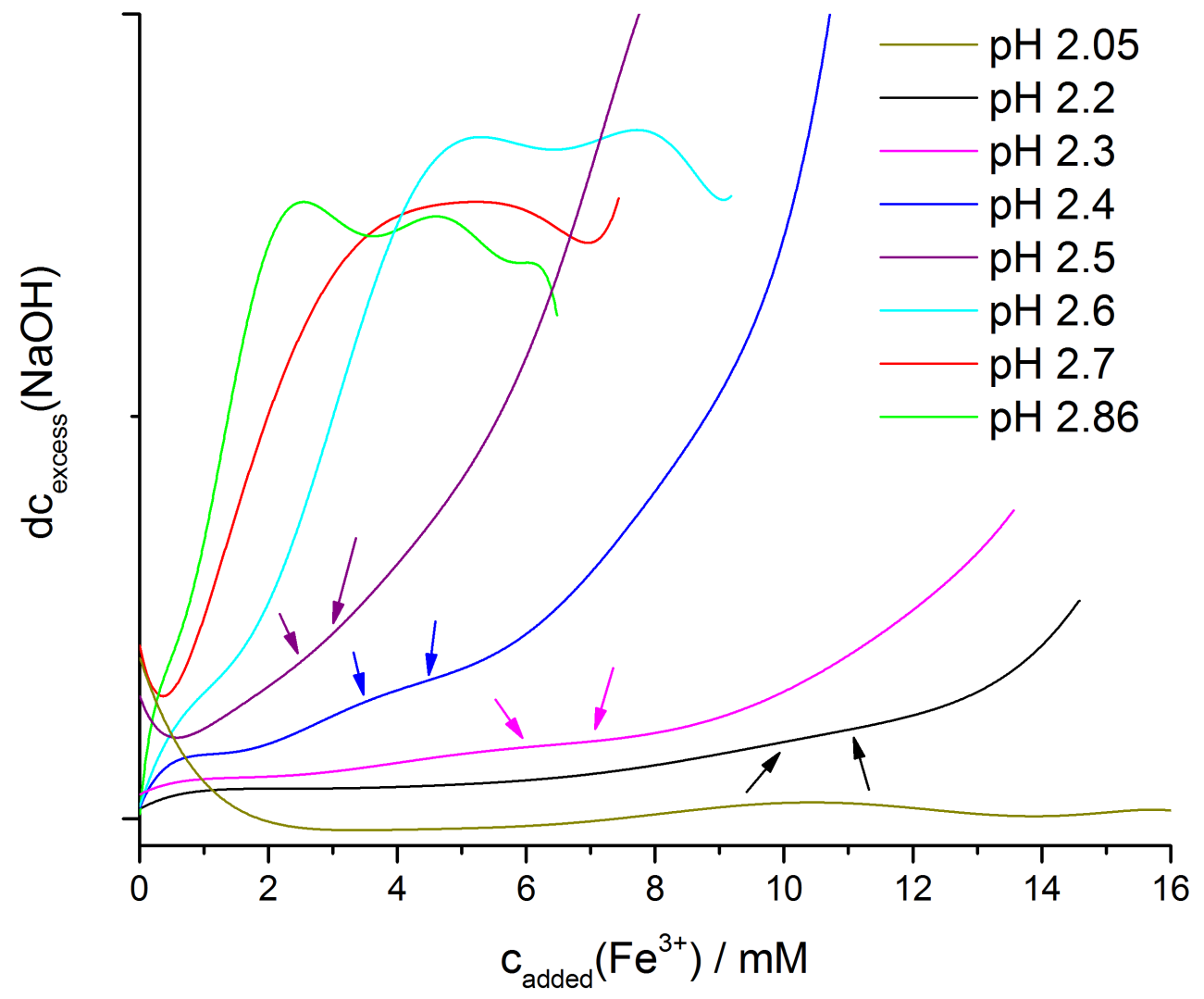

Figure S3 | Determination of the transition zone. The graphs show the smoothed derivative of a polynomial fit of the original titration data at different $\mathrm{pH}$ values. The transition zone was determined by stop experiments at $\mathrm{pH} 2.4$ (blue arrows). For the remaining $\mathrm{pH}$ values, the position of the transition region was estimated by means of the shape of the derivative function. 


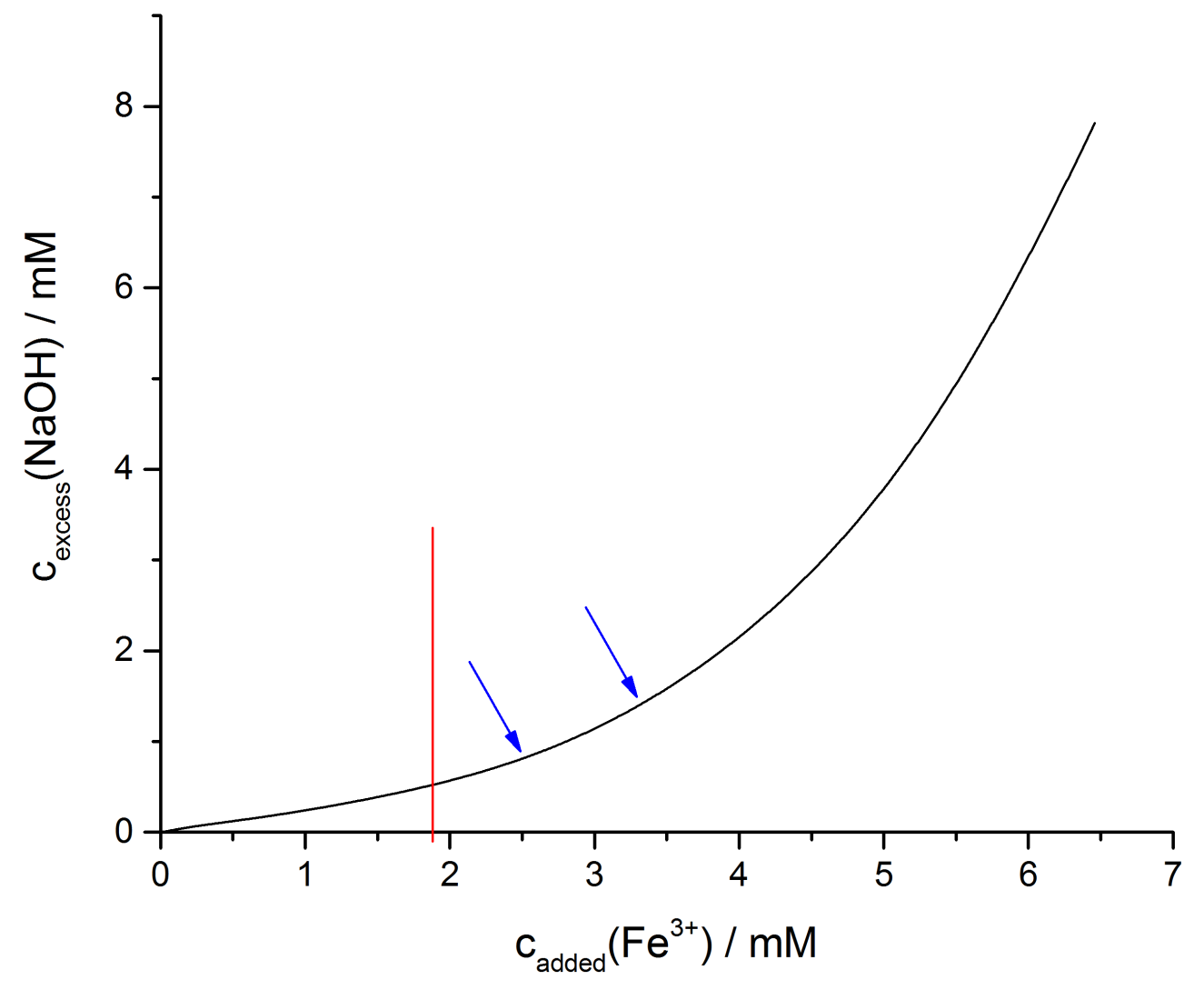

Figure S4 | Titration experiment for SAXS. The titration experiment was performed as described in the methods section at $\mathrm{pH}$ 2.4. The data was smoothed. The red vertical line depicts the transition from repulsive to aggregative behavior seen from the SAXS results. The blue arrows mark the transition region determined as described in the Supplementary Information, section 3. 

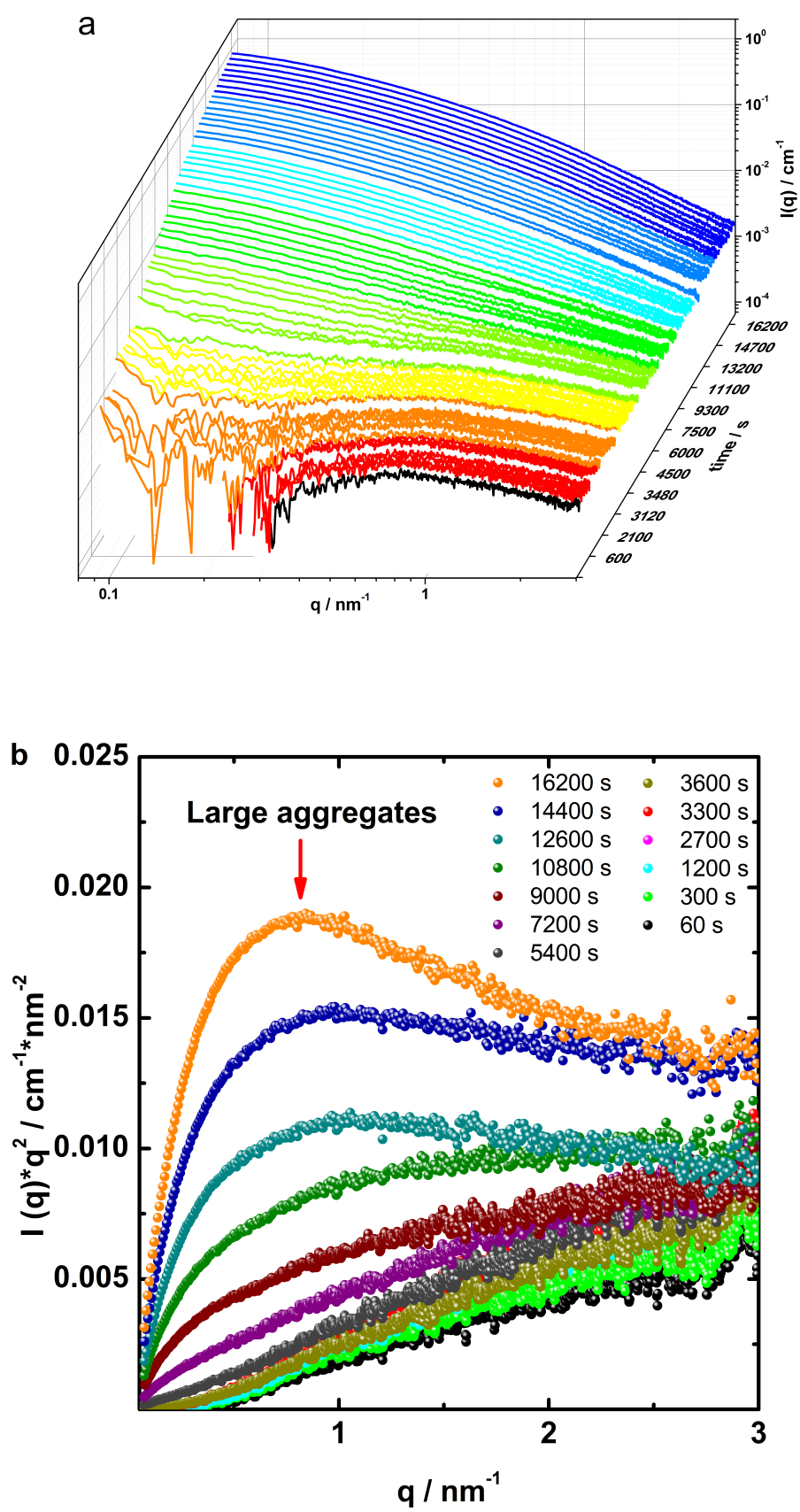

Figure S5 I SAXS curves and Kratky plots. a) Three dimensional plots of time-resolved in situ SAXS curves; b) Kratky plots of scattered intensity of $I(q) * q^{2}$ as a function of time. 

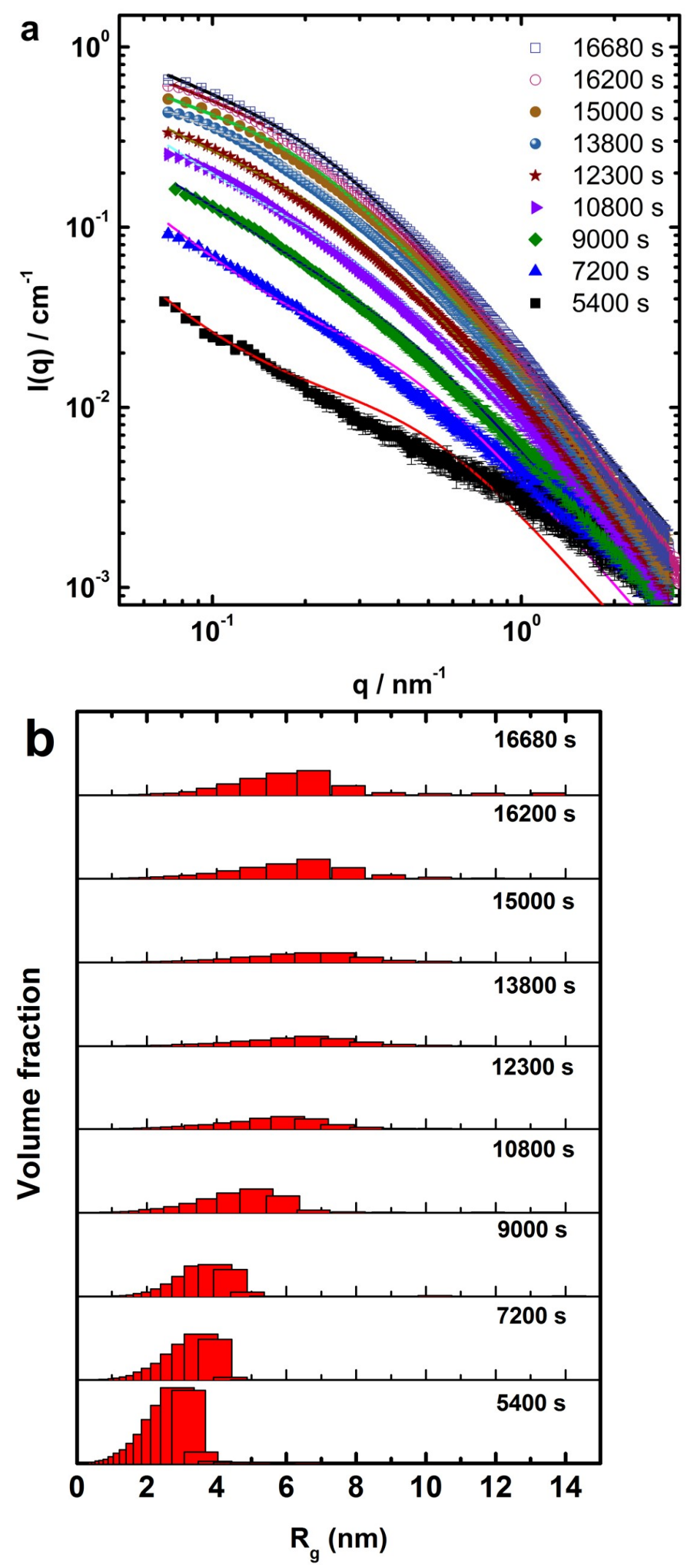

Figure S6 | (a) Best fits of scattering curves (red lines) via Monte Carlo simulation utilizing the McSAS software (see section 4 in the Supplementary Information); (b) corresponding volume-weighted radius of gyration distribution obtained from SAXS curve simulation using Monte Carlo methods. 


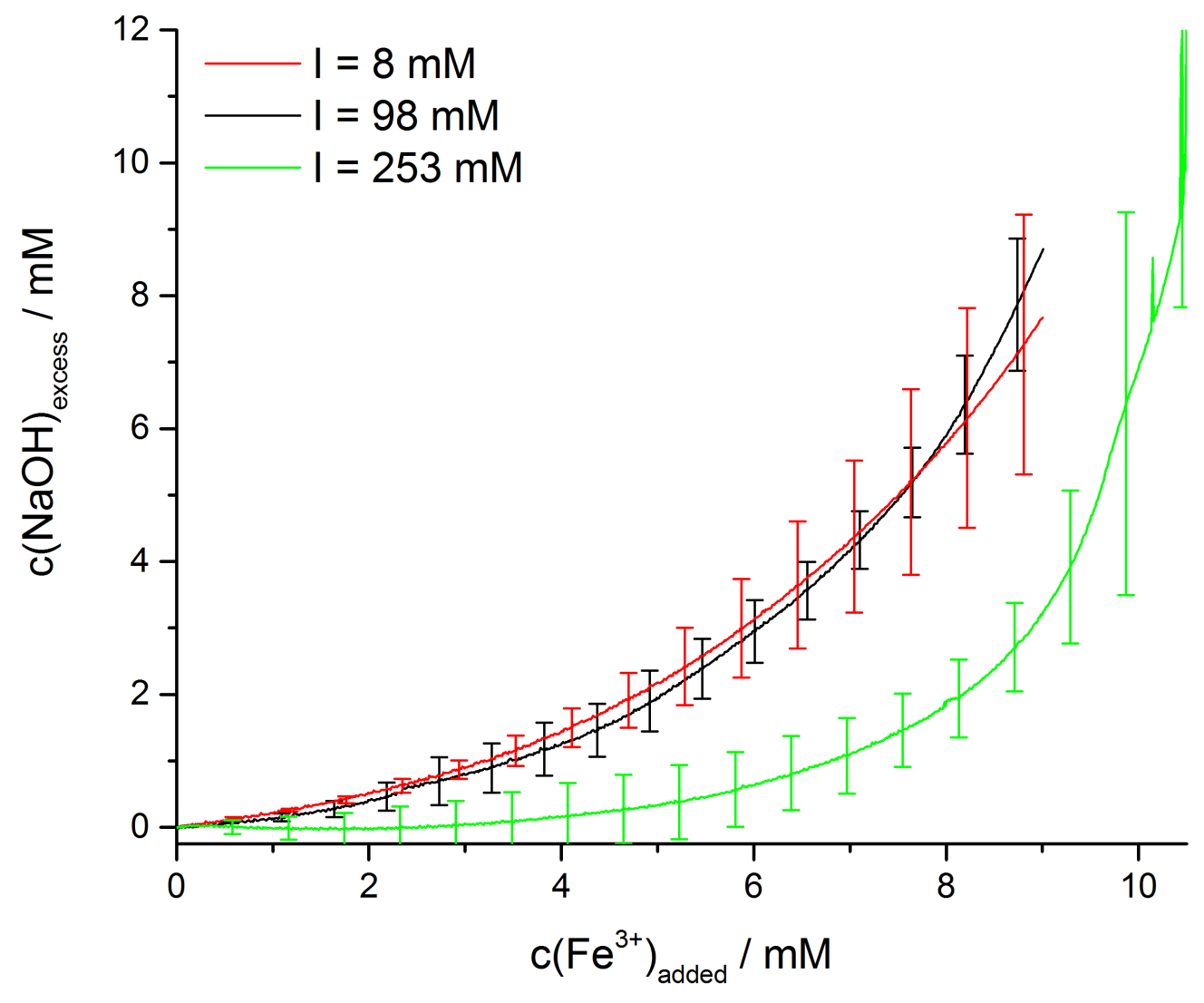

Figure S7 | Titrations at varying ionic strengths. To exclude electrostatic stabilization of the early species as reason for their non-aggregative behavior, $\mathrm{NaCl}$ was added at the beginning to increase the initial ionic strength I (as indicated) of the titration and the experiment was conducted as usual. 


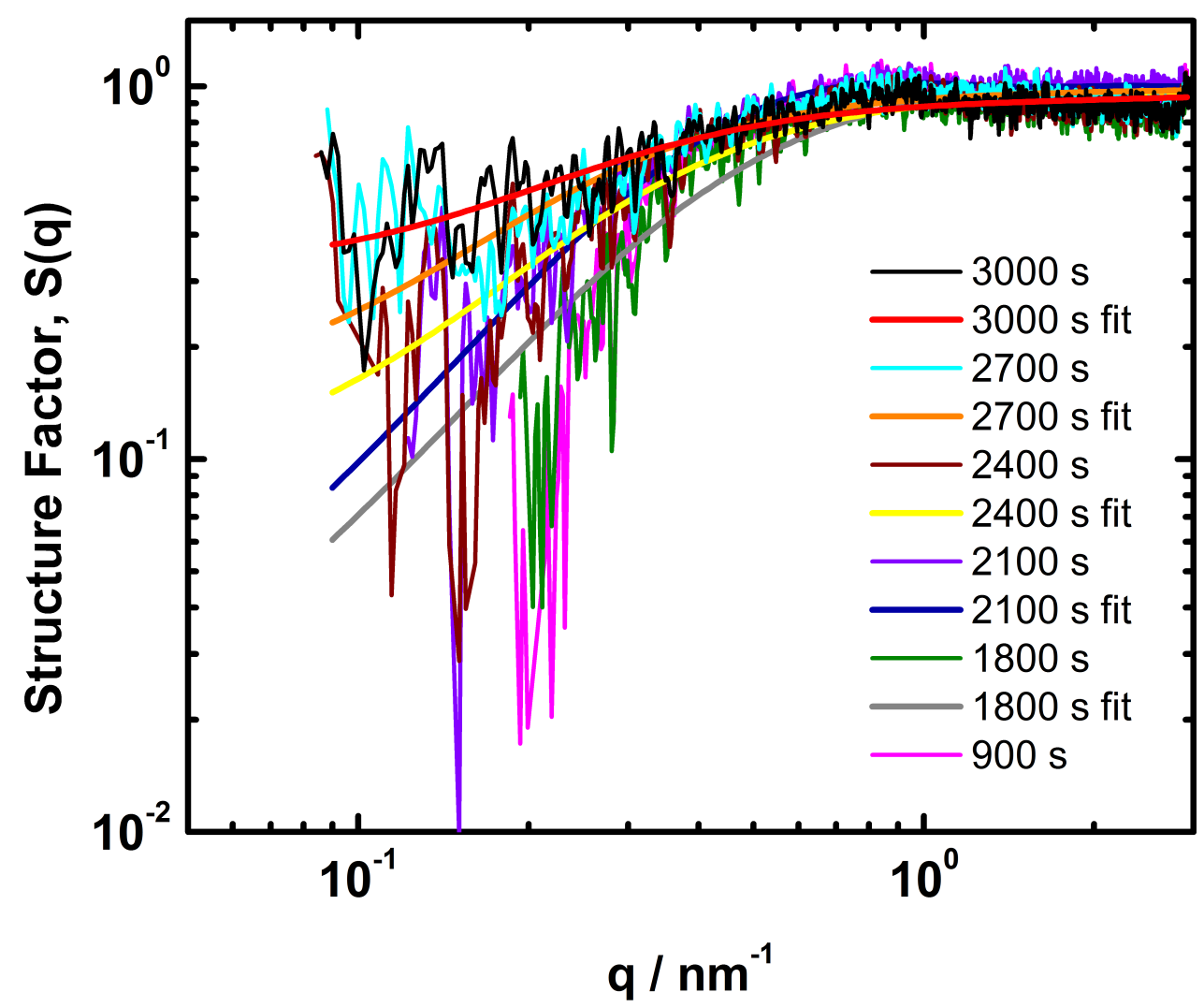

Figure S8 | Calculated repulsive structure factor, $\mathbf{S}(\mathbf{q})$. Development of $S(q)$ for different times of the reaction. The structure factor was fitted by a screened coulomb interaction potential (see section 4 in the Supplementary Information). 


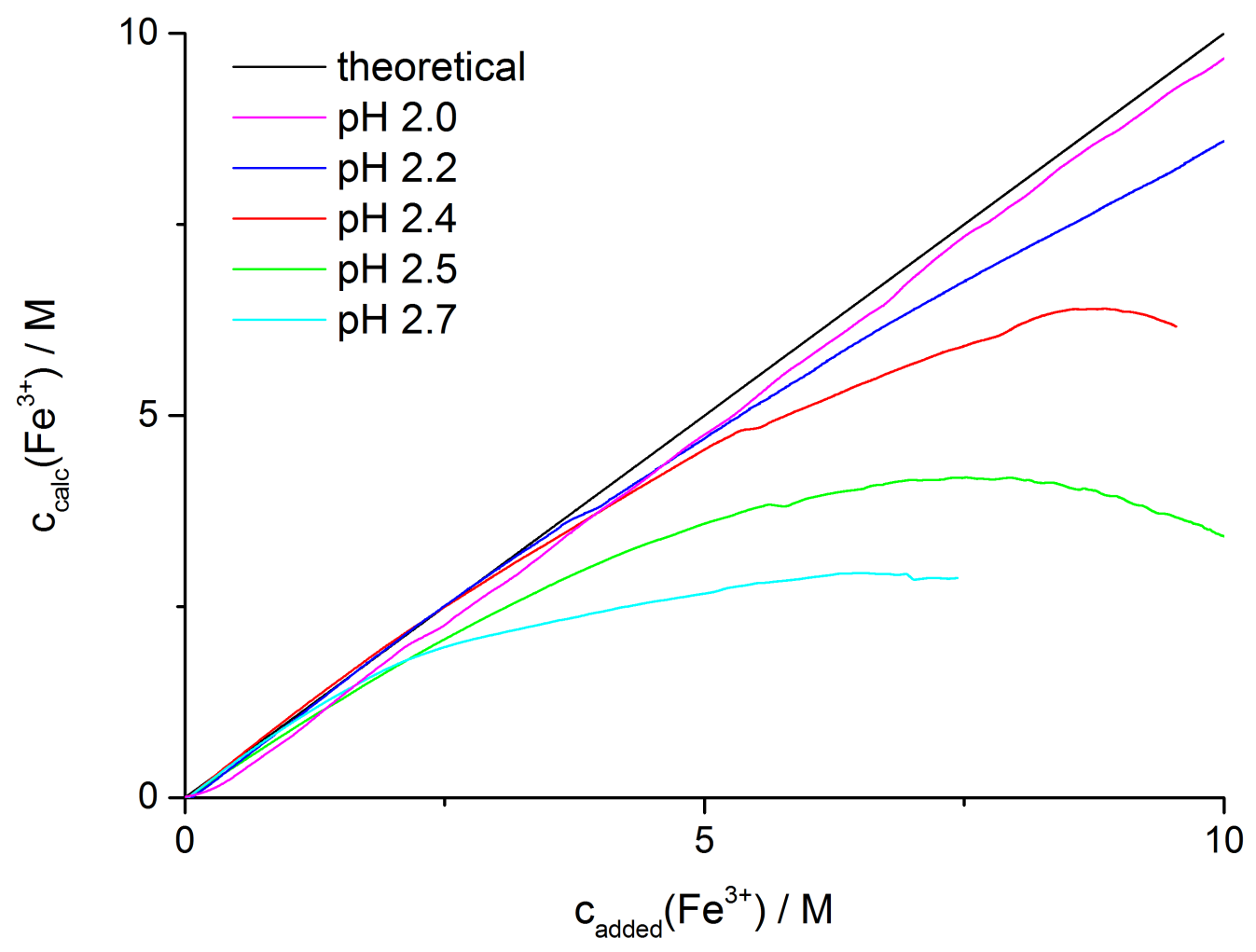

Figure S9 | Calculated concentration of dissolved iron(III) ions based on conductometry. In the beginning of the experiment measured and calculated iron concentrations match well for all investigated $\mathrm{pH}$ values. Upon the change in reaction mechanism, the concentration of free iron ions in the solution starts to deviate from the calculated value, indicating the formation of a separate phase not contributing to the conductivity of the solution. At early times and low $\mathrm{pH}$ values the obtained iron concentration exceeds the theoretical one due to electrolyte leakage. The data was smoothed. 
Table S1 | - $\log \mathrm{K}$ for the reaction of iron(III) to $\mathrm{Fe}(\mathrm{OH})^{2+}$ at different $\mathrm{pH}$ values, determined as described in literature ${ }^{2}$ and section 2 of the Supplementary Information.

\begin{tabular}{lll}
$\mathbf{p H}$ value & $\log \mathrm{K}_{\mathbf{1}}$ & $\log \mathbf{K}_{\mathbf{2}}$ \\
\hline 2 & & \\
2.2 & - & 10.91 \\
2.3 & -3.14 & 11.08 \\
2.4 & -3.17 & 11.07 \\
2.5 & -3.04 & 10.93 \\
2.6 & -2.97 & 10.89 \\
2.7 & -3.09 & 11.21 \\
2.86 & -3.00 & 11.30
\end{tabular}

Table S2 | Range of iron concentrations at which transition from olation to oxolation occurs at all investigated $\mathrm{pH}$ values

\begin{tabular}{ll}
$\mathbf{p H}$ value & $\mathbf{c}\left(\mathrm{Fe}^{3+}\right) / \mathbf{m M}$ \\
\hline 2 & \\
2.2 & - \\
2.3 & $10.0-11.0$ \\
2.4 & $6.0-7.0$ \\
2.5 & $3.6-4.5$ \\
2.6 & $2.5-3.0$ \\
2.7 & $0.8-1.5$ \\
2.86 & $0.4-0.6$ \\
& $0.3-0.5$
\end{tabular}

Table S3 | Charges and sizes of spherical scatterers obtained by fitting the repulsive structure factor employing the Hayter-MSA-Structure model (see section 4 in the Supplementary Information).

\begin{tabular}{llll} 
Time / $\mathbf{s}$ & Diameter / $\mathbf{n m}$ & Charge & lonic Strength / $\mathbf{~}$ \\
\hline & & & \\
1800 & 0.5 & $60.3 \pm 0.8$ & 0.0156 \\
2100 & 1.2 & $66.2 \pm 5$ & 0.0169 \\
2400 & 0.6 & $10.3 \pm 0.1$ & 0.018 \\
2700 & 0.52 & $6.04 \pm 0.02$ & 0.0191 \\
3000 & 0.56 & $2.02 \pm 0.02$ & 0.02
\end{tabular}

